Spatially resolved, diffuse reflectance imaging for subsurface pattern visualization toward development of a lensless imaging platform: phantom experiments

Irina Schelkanova

Aditya Pandya

Guennadi Saiko

Lidia Nacy

Hannan Babar

Duoaud Shah

Lothar Lilge

Alexandre Douplik 


\title{
Spatially resolved, diffuse reflectance imaging for subsurface pattern visualization toward development of a lensless imaging platform: phantom experiments
}

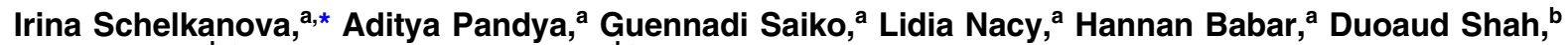 \\ Lothar Lilge, ${ }^{b, c}$ and Alexandre Douplik ${ }^{\mathrm{d}, \mathrm{e}}$ \\ ${ }^{a}$ Ryerson University, Physics Department, 60 Gould Street, Toronto, M5B 2K3, Canada \\ bUHN Microfabrication Center, Techna Institute for the Advancement of Technology for Health, Toronto M5G 2C4, Canada \\ 'University of Toronto, Princess Margaret Cancer Center, Department of Medical Biophysics, Toronto M5G 2C4, Canada \\ ${ }^{d}$ Keenan Research Center, LKS Knowledge Institute St. Michael Hospital, Toronto M5B 1W8, Canada \\ eFriedrich Alexander University Erlangen-Nuremberg, Erlangen Graduate School in Advanced Optical Technologies, Erlangen 91052, Germany
}

\begin{abstract}
A portable, spatially resolved, diffuse reflectance lensless imaging technique based on the chargecoupled device or complementary metal-oxide semiconductor sensor directly coupled to the fiber optic bundle is proposed for visualization of subsurface structures such as superficial microvasculature in the epithelium. We discuss an experimental method for emulating a lensless imaging setup via raster scanning a single fiber-optic cable over a microfluidic phantom containing periodic hemoglobin absorption contrast. To evaluate the ability of the technique to recover information about the subsurface linear structures, scattering layers formed of the Sylgard ${ }^{\circledR} 184$ Silicone Elastomer and titanium dioxide were placed atop the microfluidic phantom. Thickness of the layers ranged from 0.2 to $0.7 \mathrm{~mm}$, and the values of the reduced scattering coefficient $\left(\mu_{\mathrm{s}}^{\prime}\right)$ were between 0.85 and $4.25 \mathrm{~mm}^{-1}$. The results demonstrate that fiber-optic, lensless platform can be used for two-dimensional imaging of absorbing inclusions in diffuse reflectance mode. In these experiments, it was shown that diffuse reflectance imaging can provide sufficient spatial sampling of the phantom for differentiation of $30 \mu \mathrm{m}$ structural features of the embedded absorbing pattern inside the scattering media. ๑ 2016 Society of Photo-Optical Instrumentation Engineers (SPIE) [DOI: 10.1117/1.JBO.21.1.015004]
\end{abstract}

Keywords: microcirculation; spatially resolved reflectance; lensless imaging; microfluidic phantoms; biomedical signal detection; blood vessels; medical diagnosis; imaging.

Paper 150629PR received Sep. 24, 2015; accepted for publication Dec. 8, 2015; published online Jan. 14, 2016.

\section{Introduction}

Structural and functional analysis of superficial vasculature provides important diagnostic information about microcirculation pathogenesis. ${ }^{1}$ Dense plexus vessels participate in oxygen delivery to the dermal layer via a network of microvessels, which rise close to the surface of the epithelium and form intrapapillary capillary loops (IPCLs). ${ }^{2,3}$ In mucosal layers, the capillary network is located $\approx 250$ to $350 \mu \mathrm{m}$ below the surface. ${ }^{4,5}$ Imaging of such loops requires high spatial sampling at the depth range of $\approx 0.2$ to $0.6 \mathrm{~mm}$. Individual IPCLs are periodically distributed in the arches of the dermal papilla space. The distance between oxygenated and deoxygenated vessels of a capillary is minimal, and sometimes there is no distance at all. The mean range of distances between two successive papilla is $\approx 120 \mu \mathrm{m}$ (diffusion length of oxygen from a vessel into the interstitial tissue is about $100 \mu \mathrm{m}){ }^{2,6}$ Structural alterations of capillaries' periodicity indicate some degree of a medical condition, such as rheumatic diseases or cancer. ${ }^{6}$ Architectural disorganization, giant capillaries, hemorrhages, angiogenesis, and avascular areas characterize $>95 \%$ of patients with overt scleroderma (autoimmune rheumatic diseases). ${ }^{7}$ Dilation, elongation, and tortuousness are

*Address all correspondence to: Irina Schelkanova, E-mail: irina.schelkanova@ ryerson.ca the features of developing carcinomas. ${ }^{6,8}$ As invasion of cancer progresses, organization of the IPCLs is destroyed and the network is characterized by large vessels with no hairpin structure at the terminal branches.

There are several approaches to imaging of the superficial vascular network. Diffuse reflectance tomography (DOT) is used to evaluate tissue within a volume sampled by the light traveling from a source to a detector. ${ }^{5,9-11}$ Source-to-detector distance is one of the aspects determining the resolution of the resultant image in DOT. The technique recovers local optical properties and quantifies changes in relative concentrations of the chromophores of interest. The most traditional method of microvascular imaging is capillary magnification through a microscope or by means of cameras. This technique is termed capillaroscopy. It is mainly employed to analyze microcirculation of the vasculature from the nailfold of a finger or a toe because the scattering layer of the epidermis is very thin at those locations. ${ }^{7,12}$ When the IPCLs are located beneath thick and highly scattering epithelium layers (most of mucosa and skin), spatial architecture of capillaries is generally invisible for reflectance magnification imaging. Furthermore, capillaroscopy is highly dependent on conditions of data 
collection and quality of the imaging optics, restricting imaging to the depth of field of a thin area within a narrow field of view (FOV).

Other imaging techniques applicable for reflectance imaging of microcapillaries, such as optical coherence tomography (OCT), broadband and narrowband endoscopic imaging/spectroscopy, and intravital microscopy also suffer from various limitations. ${ }^{13}$ OCT can visualize the IPCL, but the equipment is complex, requires mechanical scanning, and is relatively expensive. ${ }^{14}$ Intravital microscopy has a limited scope for clinical applications toward human subjects. ${ }^{15}$ Overall, most of these imaging methods rely on conventional lens-based imaging approaches, which are mainly characterized by their FOVs, magnification factors, spatial resolution, and focusing. The spatial resolution and FOV are inversely proportional for the same sensor pixel density, and achieving a higher spatial resolution (through optical magnification) results in diminished FOV. Also, due to the arbitrary three-dimensional (3-D) geometries of vascular networks and living tissue motion artifacts, focusing becomes challenging under mobile conditions in-vivo. In case of microvascular pathogenesis, acquisition of large FOVs with high spatial resolutions as well as increased depth of field to study the progression of abnormal conditions is critical. Therefore, to visualize subsurface patterns effectively, a technique has to possess sufficient spatial sampling while preserving instantaneous, large FOV necessary for wide-area analysis in real time of deeply lying microvascular structures. ${ }^{16}$ Portability, reproducibility, and the ability to provide optical sectioning of depths under highly scattering conditions can be fulfilled by lensless, fiber-optic imaging platforms capable of performing high sampling with multiple source-detector geometries. ${ }^{17,18}$ Lensless imaging encompasses a broad group of applications based on a complementary metal-oxide semiconductor (CMOS)/chargecoupled device chip used for imaging of a sample with no lenses attached in front of the sensor plane to achieve a large FOV with high spatial sampling. ${ }^{19}$ This includes X-ray imaging, lensless compressive imaging, diffractive (phase) imaging, highresolution lensless endoscopy, and super-resolution imaging techniques. $^{20-24}$

The design of the fiber coupled, lens-free imaging platform proposed by our group is based on a fiber-optic plate (FOP) directly mounted onto a CMOS sensor. The FOP is placed onto, or close to, the object's surface, and reflected light is transferred to the imaging array without the use of conventional lenses. Such imaging architecture can provide a sufficiently high sampling of diffusely reflected signal and incorporate multiple source-detector configurations. Commercially available FOPs have unit fiber-core sizes of 3 to $5 \mu \mathrm{m}$ and can be tapered to achieve magnification/demagnification effects. ${ }^{25}$ At the current stage of its development, the fiber-optic, lensless imaging platform cannot demonstrate a clear advantage over lensbased systems in terms of spatial resolution and quality of the image. However, it offers a number of advantages for specific applications where diffuse reflectance imaging is valuable for its ability to recover information about attenuation of light by the tissue chromophores and where magnification is not potent.

The design described in the previous paragraph was tested and verified via performing spatially resolved imaging through raster scanning fiber-optic detectors across the absorption perturbations, under different scattering conditions and depths (thicknesses). We aimed to establish the applicability of the lensless, FOP coupled imaging sensor for diffuse reflectance imaging. To emulate a 2-D fiber-optic array arranged in a grid, a single fiber (detector) was scanned across an FOV for continuous data acquisition. Broadband illumination allowed for selection of the optimal spectral bands of wavelengths at each scan point. Through collection of sufficient statistics on parameters such as scanning step size, thickness of the scattering layers, scattering optical properties, and choice of the detector size, the method is evaluated for further development.

\section{Method}

\subsection{Spatially Resolved, Diffuse Reflectance Imaging System}

The spatially resolved, diffuse reflectance imaging setup is described in Fig. 1. Widefield, oblique collimated illumination of the entire sample was achieved with a $0.40-\mathrm{mm}$ core optical fiber (Silica fiber, NA 0.22 , Thorlabs Inc.) coupled to a broadband white light source, HPLS-30-02 (Thorlabs Inc.), and a fiber optic collimator (OZ Optics, Canada). For image acquisition, regions of interest of $1 \mathrm{~mm} \times 0.060 \mathrm{~mm}$ and $0.50 \mathrm{~mm} \times$ $0.050 \mathrm{~mm}$ were chosen to minimize the time of mechanical
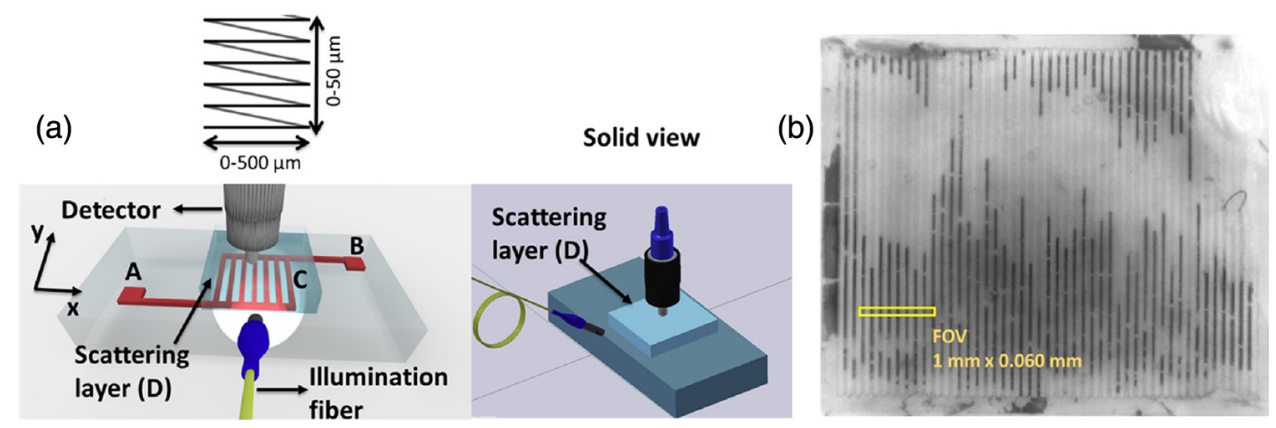

Fig. 1 (a) The experimental setup illustrates the raster scan setup for collection of the reflectance signal from the phantom with microchannels (area $\mathrm{C}$ ) filled with hemoglobin through reservoirs $\mathrm{A}$ and $\mathrm{B}$. Optical fiber was raster scanned in a pattern depicted above. The y step size was kept constant at $5 \mu \mathrm{m}$, and the $\mathbf{x}$ step size was varied while keeping a constant field of view (FOV). The illuminating fiber provides oblique, collimated illumination (angle was kept close to 45 deg with the $\mathbf{y}$ axis pointing downward) of the microfluidic phantom covered by scattering layers $(D)$ of different thicknesses and optical properties. The detector fiber then transfers the reflected photons to the spectrometer. (b) Image of microfluidic platform taken with $10 \times$ objective at $\approx$ unit magnification. The FOV for the scanning experiments with $10-\mu \mathrm{m}$ core fiber is outlined by the rectangle $\left(1 \times 0.060 \mathrm{~mm}^{2}\right)$. The area of microfluidic is $5.75 \times 5 \mathrm{~mm}^{2}$. 
Table 1 Physical values of the $\mu_{\mathrm{s}}^{\prime}\left(\mathrm{mm}^{-1}\right)$ at $\lambda=545 \mathrm{~nm}$ for a range of $\mathrm{TiO}_{2}$ concentrations (columns), and thicknesses (rows) of the upper diffusive layers. Thus, B2 will be the layer with $\mu_{\mathrm{s}}^{\prime}=1.564 \mathrm{~mm}^{-1}$ and thickness of $0.32 \mathrm{~mm}$.

\begin{tabular}{|c|c|c|c|c|c|c|}
\hline \multirow{3}{*}{\multicolumn{2}{|c|}{ Thickness $(\mathrm{mm})$}} & \multicolumn{5}{|c|}{ Scattering coefficient $\left[\mu_{\mathrm{s}}^{\prime}\left(\mathrm{mm}^{-1}\right)\right]$} \\
\hline & & \multirow{2}{*}{$\frac{A}{0.01 \%}$} & \multirow{2}{*}{$\frac{B}{0.01 \%}$} & \multirow{2}{*}{$\frac{C}{0.02 \%}$} & \multirow{2}{*}{$\frac{D}{0.02 \%}$} & \multirow{2}{*}{$\frac{E}{0.03 \%}$} \\
\hline & & & & & & \\
\hline 1 & $0.23 \pm 0.021$ & 0.894 & 1.391 & 1.904 & 3.216 & 3.714 \\
\hline 2 & $0.32 \pm 0.012$ & 0.846 & 1.564 & 2.43 & 3.215 & 4.177 \\
\hline 3 & $0.36 \pm 0.015$ & 0.993 & 1.474 & 2.99 & 3.265 & 4.248 \\
\hline 4 & $0.62 \pm 0.014$ & 0.927 & 1.922 & 2.749 & 2.663 & 3.479 \\
\hline 5 & $0.73 \pm 0.078$ & 0.877 & 1.589 & 2.067 & 2.106 & 3.369 \\
\hline
\end{tabular}

scanning. Two individual optical fibers were selected to collect the reflected signal: a $10-\mu$ m multimode silica detector fiber (NA 0.1 , Thorlabs Inc.), and a $50 \mu \mathrm{m}$ (NA 0.22, Thorlabs Inc.). One fiber at a time was connected to a spectrometer (Ocean Optics USB4000), which had a spectral range of 200 to $1100 \mathrm{~nm}$ with a resolution of 1.5 to $2.3 \mathrm{~nm}$ full width at half maximum. Integration time range was between 5 and $200 \mathrm{~ms}$. The spectrometer data were processed using a custom script in MATLAB (MathWorks) written in our laboratory.

For comparison with the traditional lens-based setup, several images were acquired with an RGB CMOS chip (Motic, China) using a $10 \times$ objective $(0.25 \mathrm{NA}$, Motic, China). The sensor was placed close to the back focal plane of the objective, permitting a magnification ratio of $\sim 1$. The CMOS matrix had physical dimensions of $6.8 \mathrm{~mm} \times 5.3 \mathrm{~mm}$ and a maximum resolution of 3 megapixels (MP; $2048 \times 1536$ ), which corresponds to a pixel pitch $\approx 3.2 \mu \mathrm{m}$, neglecting packaging area losses at the chip boundary. Spectral resolution of the CMOS was estimated as the spectral width of the red-green-blue channels $(\approx 100 \mathrm{~nm})$. The Software Motic Images Plus 2.0 for PC software package was used for image acquisition, and the images had a digital resolution of 8 bits. The visualization of images and the image processing were performed by an algorithm created in MATLAB (MathWorks).

The microfluidic platform is composed of narrow channels covered with various scattering layers (prepared separately) placed on the top (D in Fig. 1). The microchannel plate was manufactured from a clear polydimethylsiloxane (PDMS, silicone) material; $n=1.56$ in the visible spectrum without scattering particles. The parallel grooves were designed to approximate a nailfold microvascular spatial pattern with mean physical dimensions: $34-\mu \mathrm{m}$ wide groove, separated by $82-\mu \mathrm{m}$ spacing, and a groove height of $30 \mu \mathrm{m} .^{2,26}$ The solution of the $90 \%$ to $95 \%$ oxygenated, human hemoglobin (Agat-Med, Russia, $160 \mathrm{~g} / \mathrm{l}$ ) was injected in reservoir A in Fig. $1(2 \mathrm{~mm} \times 2 \mathrm{~mm})$ by means of 18 -gauge needle syringes. Hemoglobin was then redistributed from reservoir A to reservoir B $(2 \mathrm{~mm} \times 2 \mathrm{~mm})$ to obtain a close to uniform distribution of hemoglobin in the microfluidic channels (area $\mathrm{C}$ in Fig. 1). (a)

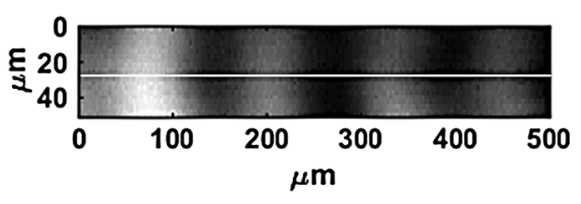

(c)

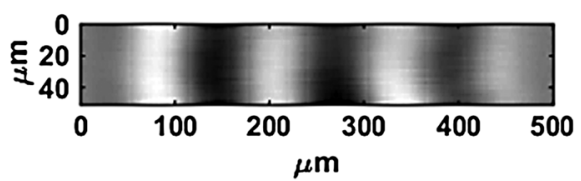

(b)

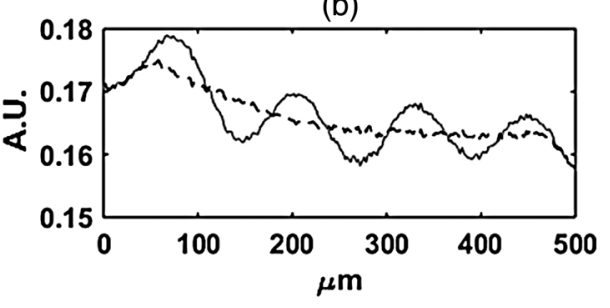

(d)

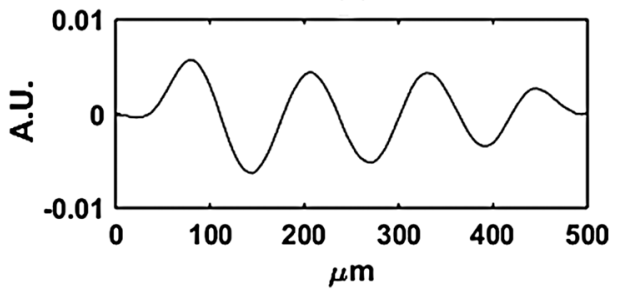

Fig. 2 Image processing steps: (a) a raw reflectance image formed through the raster scan is shown. (b) A line plot of the intensity variation across the white line at $25 \mu \mathrm{m}$ in (a). The dashed line in plot (b) represents a moving average filter with a kernel size of half the scan line width emulating the background variation in light intensity due to the illumination variance. (d) A background corrected version of the scan line and (c) corresponding background corrected image. 


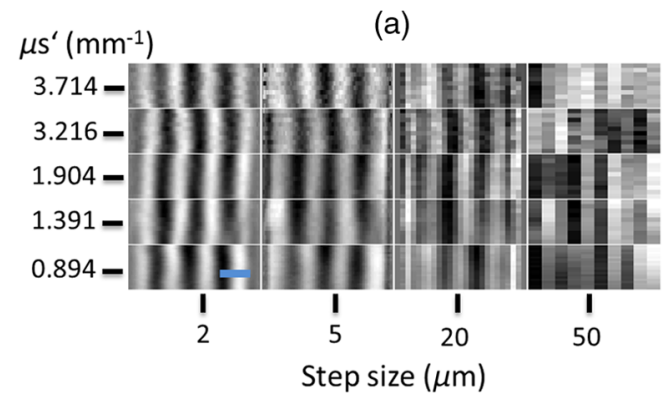

(b)

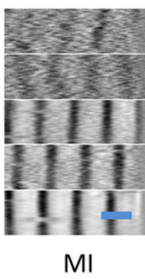

Fig. 3 Grayscale images of the microfluidic platform for a fixed thickness $(d=0.24 \mathrm{~mm})$, different scattering properties of the upper layer at $\lambda=545 \mathrm{~nm}$. The scanning step (from left to right) tested for $50 \mu \mathrm{m}$ detector core (a), FOV $=0.05 \times 0.5 \mathrm{~mm}^{2}$ is $2,5,20,50 \mu \mathrm{m}$ and (b) $10 \times$ magnification images of the same scattering layers acquired with microscope camera. The horizontal bars on the bottom right corner of the images represent $100 \mu \mathrm{m}$.

\subsection{Fabrication of Top Scattering Layers}

To approximate the optical properties of biotissue, the microfluidic channels were covered by layers of variable thickness and scattering properties, produced with Sylgard ${ }^{\circledR} 184$ Silicone Elastomer DOW/Corning, a two-component silicone product with a refractive index of 1.41. This silicone allows construction of complex geometries and easy incorporation of scatterers. The scatterer concentrations were based on weight percentage of $\mathrm{TiO}_{2}$ (Sigma Aldrich) reported in the literature. About $0.005 \%$, $0.010 \%, 0.015 \%, 0.020 \%$, and $0.025 \%$ concentrations were selected to match the scattering properties $\left(\mu_{\mathrm{s}}^{\prime}, \mathrm{mm}^{-1}\right)$ of the epithelium. ${ }^{27-29}$ The geometric thickness of the layers was measured with a high-precision gauge. The results were found to be consistent with similar silicone phantom thickness measurements reported in Ref. 30. The averaged thickness of the silicone (a)

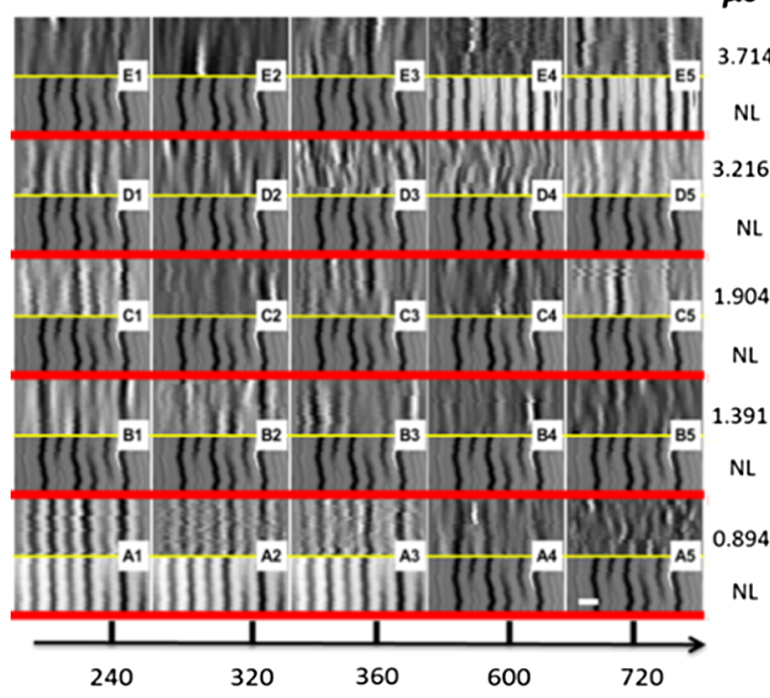

$\mu \mathbf{s}^{\prime}$

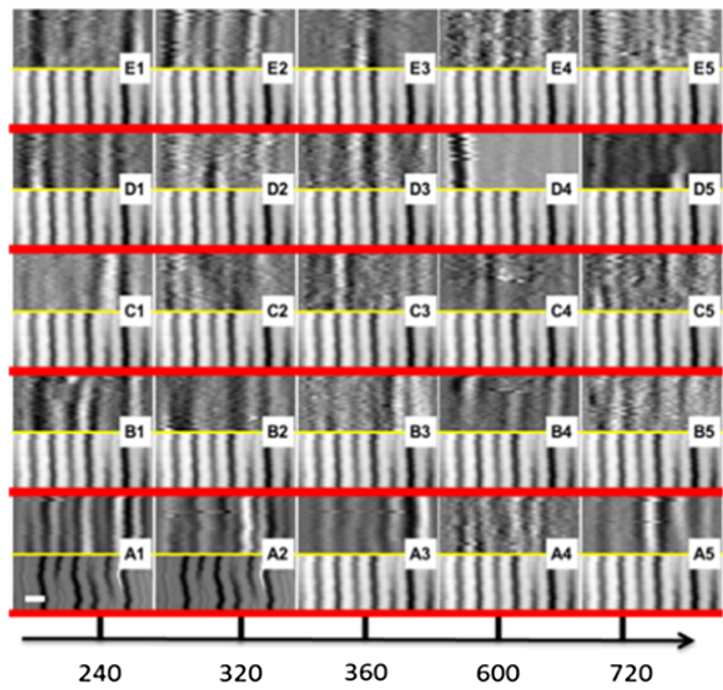

(c)
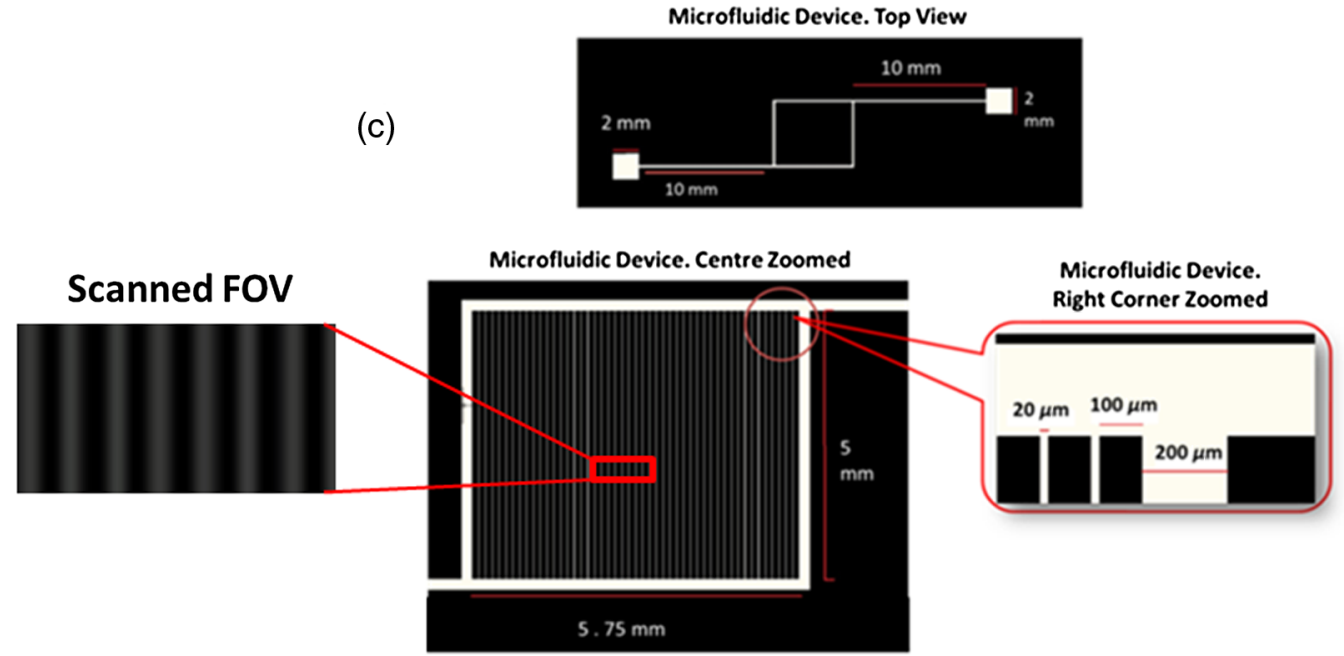

Fig. 4 (a) Images for five thicknesses (x-axis) and five scattering properties (y-axis) scanned with $10 \mu \mathrm{m}$ core fiber $(\mathrm{NA}=0.1)$ are presented. The red line demarcates scattering properties, the yellow line divides one image into two halves: lower part (NL: no layer) is an image scanned without any scattering layer from the same location as the image collected via a top layer (image above the yellow line). (b) The same experiments were repeated for $50-\mu \mathrm{m}$ core fiber detector. Scanning step is $2 \mu \mathrm{m}$. (c) The layout of the microfluidic phantom and the corresponding scanned FOV. The white channels in the microfluidic are the dark channels in the scanned images ( $a$ and $b$ ) as these channels were filled with hemoglobin. 
layers with standard deviation and their scattering properties are given in Table 1.

Individual scattering blocks were made by mixing the desired concentration of scatterer with the curing agent component of the silicone elastomer. To obtain a homogeneous mixture, it was placed in an ultrasonic bath (Branson, 1510R-DTH) for $10 \mathrm{~min}$ at $30 \mathrm{kHz}$ to break residual clusters. The curing agent was then mixed with the silicone (1:9 weight ratio) under careful stirring. Remaining air bubbles were removed by using a vacuum pump (Barnant) that kept the mixture under low pressure for $30 \mathrm{~min}$. A small portion $(0.5 \mathrm{ml})$ of the final mixture was placed between two standard microscopic glass slides, separated by two spacers within the range of 0.200 to $0.800 \mathrm{~mm}$. Finally, a thin single-layer scattering phantom block was produced by curing at $30^{\circ} \mathrm{C}$ for $6 \mathrm{~h}$ or at room temperature for $24 \mathrm{~h}$. Then this block was gently placed over the microfluidic channel area before starting measurements. Electrostatic forces kept the phantom scattering layer and the microfluidic together. To verify the scattering properties of the phantom blocks in the visible range (400 to $700 \mathrm{~nm}$ ), the integrating sphere technique (UV-VIS-NIR Spectrophotometer Shimadzu UV-3600, Japan) was used. An inverse adding doubling software program was used to compute the optical properties of the samples. ${ }^{31}$

\subsection{Image Processing}

Spectral data acquired at each point resulted in a dataset of the dimensions $\quad\{x, y$, wavelengths $\}=\{500(1000) \mu \mathrm{m} / x$ step size, $50(60) \mu \mathrm{m} / \boldsymbol{y}$ step size, 3648 wavelengths $\}$. The raster scan was performed for $\boldsymbol{x}$ and $\boldsymbol{y}$ step sizes of 2, 5, 10, 20, and $50 \mu \mathrm{m}$, for two FOVs $0.50 \times 0.050 \mathrm{~mm}^{2}$ and $1 \times 0.60 \mathrm{~mm}^{2}$, as shown in Fig. 1. For choosing a spectral band with the highest image contrast, spatial variance analysis was performed on a dataset obtained without any scattering layers. The spectra were divided into $10-\mathrm{nm}$ bands across 450 to $800 \mathrm{~nm}$. The mean of each band was used to calculate the normalized variance in the spatial dimensions. Before calculating variance, the data were mean centered and normalized to the mean.

Due to the oblique setting of light, background intensity varied across the FOV. In order to account for variations, we assumed that the fluctuations in the background illumination were of low spatial frequency compared to the fluctuations exhibited by the embedded hemoglobin contrast obtained from the microfluidic platform. As shown in Fig. 2(b) (smoothed version denoted by dashed line), a lowpass filter was employed with an adaptive moving average kernel having a size $(N)$ [Eq. (2)] of half the scan line length in the $x$ direction of the image. The filtered image $\left(I_{\operatorname{avg}(x, y)}\right)$ was then subtracted from the original image $I_{(x, y)}$ [Eq. (1)]. This resulted in an image with background correction [Eq. (3)], showing pure contrast of the microfluidic channels filled by hemoglobin. Because the scan was performed line by line and an image was formed by a collection of such lines, the moving average filter was estimated to correct for the background for each line.

$I(x, y)=\left[\begin{array}{ccc}I_{11} & \cdots & I_{x 1} \\ \vdots & \ddots & \vdots \\ I_{1 y} & \cdots & I_{x y}\end{array}\right]$,

$$
I_{\mathrm{avg}}(i, y)=\frac{1}{N}\left(I_{i-\frac{N}{2}, y}+I_{i-\frac{N}{2}+1, y}+\ldots \ldots \ldots I_{i-\frac{N}{2}+N, y}\right)
$$

where $i=1$ to $x$, for a linear scan, $y=1$

$I_{\text {background }_{\text {subtract }}}(x, y)=I(x, y)-I_{\text {avg }}(x, y)$.

\section{Results}

The alternating dark and light bands observed on all images represent an absorption contrast within narrow FOVs after the light has traveled through the hemoglobin grooves of the phantom under a scattering layer [Fig. 1(b)]. Properties of all diffusive layers for a selected wavelength, $\lambda=545 \mathrm{~nm}$, are listed in Table 1. For labeling purposes, scattering properties of the layers were coded with letters A to E and thickness was coded from 1 to 5. All together, five thickness and five scattering coefficients were tested experimentally, resulting in 25 datasets for image analysis. Images were presented with a modified aspect ratio in order to better visualize the patterns.

Dependence of image resolution on the scanning step size, thickness of the upper layers, scattering property, and detector size can be observed in Figs. 3-5. Quality of an image was significantly affected by the scanning step for the $50-\mu \mathrm{m}$ detector (Fig. 3). Images formed from 2- $\mu \mathrm{m}$ step size scans demonstrated superior contrast despite the interrogated volume sampled by the fairly large detector diameter. As anticipated, increasing the reduced scattering coefficient and scanning step size decreased the quality of the image due to blurring from "A1-2" (least scattering and scanning step size) to "E1-50" (largest scattering and scanning step size).

To compare deterioration of the optical signal due to the thickness ( $\mu \mathrm{m}$, from left to right) and increased scattering property of the layers $\left[\mu_{\mathrm{s}}^{\prime}\left(\mathrm{mm}^{-1}\right)\right.$ from bottom to top], the matrix of the images with all 25 experimental conditions is displayed in Fig. 4 for (a) $10-\mu \mathrm{m}$ and (b) $50-\mu \mathrm{m}$ detectors. Analyzing the contrast for the scanning step of $2 \mu \mathrm{m}$, we observe that decreasing the step size (i.e., a detector size) can dramatically increase the imaging quality (at least for linear objects) due to high

(a) $\mu \mathrm{s}^{\mathrm{c}}\left(\mathrm{mm}^{-1}\right)$

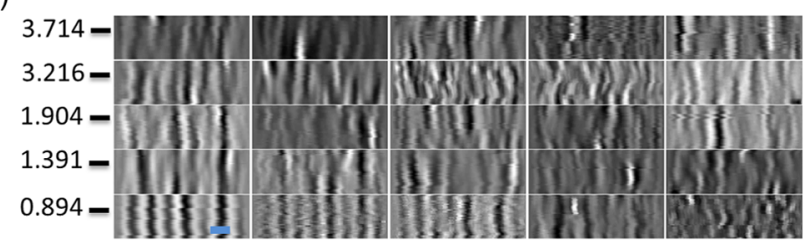

(b)

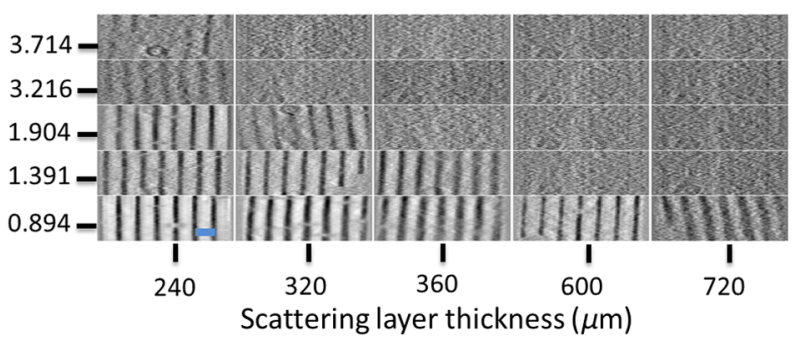

Fig. 5 (a) Scanned FOV $\left(1 \times 0.06 \mathrm{~mm}^{2}\right)$ diffuse reflectance images for all thicknesses ( $x$-axis) and (b) all scattering ( $y$-axis) acquired with $10-\mu \mathrm{m}$ core fiber, $2-\mu \mathrm{m}$ step size are compared with 10x magnification images. Preprocessing algorithm is same for (a) and (b). The small bars inset in the bottom left of the figure represent $100 \mu \mathrm{m}$. 
spatial sampling (Fig. 3). Also, to ensure that the observed pattern of dark lines is due to the absorption by the hemoglobin in the channels and not an image-processing artifact, two images of no-layer scan and scan through the scattering layer were performed on exactly the same location. The images obtained after scanning with a scattering layer were aligned with its corresponding no-layer scan (Fig. 4).

Diffuse reflectance images collected with both detectors were compared with $10 \times$ magnification images of a conventional microscope (Figs. 3, 5, and 6). For every matching condition, magnification images were put through the same image processing algorithm as for the scanned figures. Significant loss of visibility of the absorption pattern occurred for lens-based

(a)

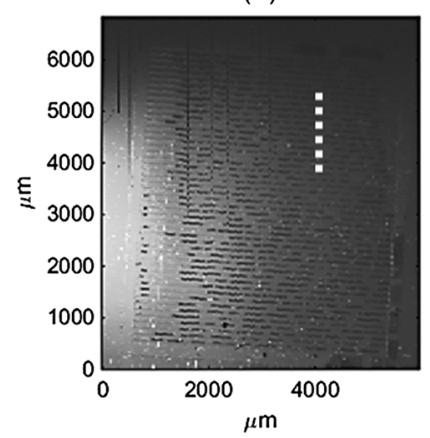

(c)

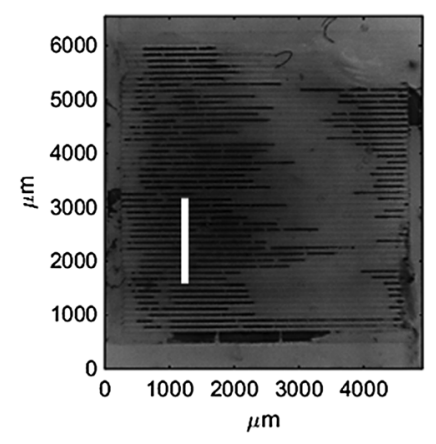

(e)
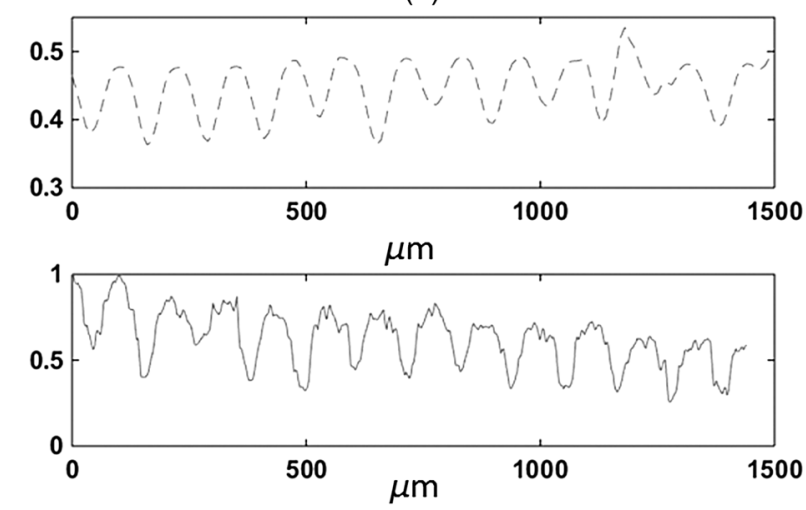

Fig. 6 Fiber scanned images of large FOVs obtained with two detectors: (a) $10-\mu \mathrm{m}$ fiber with scanning step $=10 \mu \mathrm{m} \times 50 \mu \mathrm{m}$ and (b) $50-\mu \mathrm{m}$ fiber with scanning step $=10 \mu \mathrm{m} \times 20 \mu \mathrm{m}$ are compared with lens-based images: (c) 10x magnification objective of the microscope, and (d) a 50-mm planoconvex lens. Amplitude of the signal for both ( $a$ and $c$ ) conditions are plotted in (e). Dashed lines represent a line extracted from (a), and the solid line indicates a line extracted from (c). No scattering layers were present.
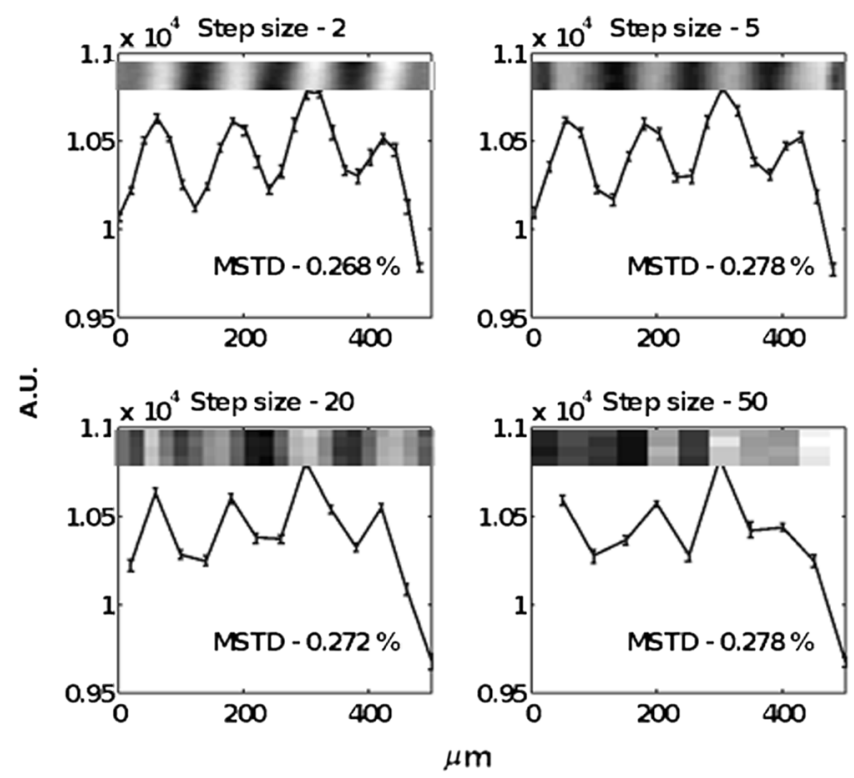

Fig. 7 The plot shows standard deviation for a sample size of 10 trials for different scan step sizes. The FOV and the scattering layer were kept constant for all these trials and the mean standard deviation (MSTD) shows low signal variability. The insets represent the scanned image corresponding to the structure of the periodic absorbing channels.

images for a higher scattering property of the layers as well as for the deeper-located sample.

Analysis of the amplitude of the optical signal from the scanning experiments and lens-based images are compared in Fig. 6. The 2-D image of the microfluidic platform represents a pattern of periodically absorbing bands embedded in the transparent background of the PDMS. Although it is expected that the image of parallel channels should yield the shape of the "rect" function, the manufacturing of the microfluidic and distribution of absorbers inside the grooves indicates that the shape of the amplitude is contaminated and deviates from the square amplitude of the "rect" function, even for the surface image of the $10 \times$ objective image [Fig. 6(e)]. In case of diffusive media, an ideal shape of the optical signal is traded for recovery of the attenuation amplitude from the scattering subsurface of the sample, where an advantage of the absorption-based method is evident (Figs. 5 and 6). Although the images obtained through scanning show distortions, it is estimated that an optomechanical system composed of a multifiber assembly with an instant large FOV image collection would get rid of distortions generated due to the uncertainty of the scanning stage.

To evaluate the reproducibility of images and stability of the experimental setup, we performed 10 repeated trials of raster scans on layer A3 (Table 1). The resultant mean and standard deviation are shown in Fig. 7. The interexperiment variability appears to be quite low and is presumably caused mainly by uncertainty of placement of the fiber by the $x-y$ stage $(0.5 \mu \mathrm{m})$ and temporal fluctuations of light intensity. Thus, major variations in the diffuse reflectance signal can be mostly attributed to the absorption contrast by the hemoglobin channels of the microfluidic platform.

\section{Discussion and Conclusions}

Imaging of microvasculature in vivo is one of the core research directions in the diffuse reflectance imaging field. The main 
limiting factor of diffuse reflectance imaging is the scattering in biological tissues, which decreases image contrast and restricts the sampling depth. Diffuse optical tomography is a process of mapping local optical properties of tissues with multiple sourcedetector configurations and reconstruction of the attenuation coefficients by means of mathematical algorithms. The resultant spatial sampling is quite poor, and the need to improve resolution of microvascular images persists. ${ }^{11,18}$ We propose a method to overcome some of the limitations of diffuse reflectance imaging by employing the latest advances in sensor and fiber-optic technologies. In this study, a fiber-optic scanning system was employed to collect spatially resolved diffuse reflectance images from a phantom for testing technical parameters for a design of an imaging architecture. Overall, the results have shown that sampling microscale subsurface patterns with fiber optics can provide a low-cost solution for clinical imaging through scattering media.

As a proof of principle of the device, it was demonstrated that images formed via mechanical scanning of the pattern maintain intensity variations of the absorbing structure through scattering layers of up to $\mu_{\mathrm{s}}^{\prime}=4 \mathrm{~mm}^{-1}$ and $0.72 \mathrm{~mm}$ thick (Figs. 3-5). Selection of the diameters of the detector-fibers was based on the physical dimensions of the microfluidic phantom: an individual channel of $30-\mu \mathrm{m}$ width has sufficient sampling points to be detected with a $10-\mu \mathrm{m}$ detector, whereas the entire period between two neighboring grooves is probed by a $50-\mu \mathrm{m}$ detector. According to the Nyquist theorem, dimensions of the detectors and step sizes are within the limits of necessary sampling and should not show any image aliasing. Even so, the mechanical scanning system was set for overlapping sampling of the region of interest for better visualization of absorption contrast. While keeping the area of interrogation constant, we collected light in virtual subpixel arrangements to compensate potentially limited sampling of the detectors and to confirm that the experiments were not affected by spatial frequency artifacts. This is especially evident from a full FOV figure of the entire microfluidic [Figs. 6(a) and 6(b)], where one can see all the features of the phantom as compared with the lens-based images.

Traditional diffuse reflectance imaging systems suffer from low resolution $(\sim \mathrm{mm}) /$ large sampling volumes due to the source-detector configurations of the probes. ${ }^{11}$ Resolution of our fiber-optic-based system is the function of an individual core dimension of the FOP and the interrogation volume $\left(\lambda, \mu_{\mathrm{s}}^{\prime}\right)$; i.e., the smaller the core of a fiber, the closer the source-detector distance and the higher the sampling. Although further minimization of fiber core (detector) size results in diminished light collection efficiency, leading to longer integration times, it can be alleviated by exploiting high sensitivity sensors (e.g., electron multiplying charge coupled devices) and by binning the pixels (similar to mosaic pattern binning of RGB images). As an example, modern endoscopes rely on fiberoptic image conduits with small cores $(\sim 2$ to $5 \mu \mathrm{m})$ for deep cavity imaging. We built our prototype to operate under similar light intensity levels.

In order to verify the performance of our lensless, fiber-optic approach against standard lens-based imaging, we acquired reflectance images of the microfluidic phantom with scattering layers using a $10 \times(0.25 \mathrm{NA})$ microscopic objective and a $50-\mathrm{mm}$ planoconvex (0.26NA) lens (Figs. 3, 5, and 6). Figure 5(b) depicts limits of contrast visibility that demonstrates the diminishing capability of a lens-based system to image contrast under higher scattering and larger depths, whereas the lens-free imaging technique performs better at recovering absorption contrasts under similar conditions. Although focusing errors of the lensbased setup can be overcome by physical scan of the focal spot and stitching together the obtained images to gather a large depth of field (and FOV), it still requires mechanical motion, and for a portable device, it limits practicality and raises the cost. To emphasize, the proposed method of fiber-optic imaging is not in direct competition with the lens-based technologies in terms of spatial sampling; rather, it can offer a focus-independent interrogation depth for a specific clinical demand. In particular, it can be used to assess optical density of the epidermal layer (to estimate its thickness) and calculate density of IPCLs and their pattern periodicities, especially in case of horizontal capillary loops, which are close to surface (e.g., nailfold, lips, etc.). Furthermore, it is advantageous under mobile conditions as it is not affected by loss of focusing on uneven surfaces, collects spectral information, and has a sampling depth that can be adjusted by implementing narrow-band imaging. By contrast, any lens-based approach requires a working distance between the image and object planes governed by the optics used. Typically, this is an unwanted trait when designing portable point-of-care devices, which need to have a small form factor (e.g., a device the size of a pulse oximeter). Finally, it has been found that lensless imaging is able to detect absorption inhomogeneities on a depth of more than one transport mean free path $\left(1 / \mu_{\mathrm{s}}^{\prime}\right)$, which outperforms lens-based systems in our previous estimates. ${ }^{32}$

In summary, imaging of subsurface linear patterns through a diffusive environment based on the absorption contrast was demonstrated using a fiber-optic scanning system. Spatially resolved diffuse reflectance images collected from the microfluidic phantom demonstrate that the fiber-optic imaging method can provide sufficient sampling of microstructures to recover information about subsurface lines as small as $30 \mu \mathrm{m}$. Such an imaging architecture was tested as a proof of principle experiment toward the development of a lensless fiber-optic imaging platform. The compact design of the FOP-based camera can be further improved with implementation of smaller diameter core fibers and smaller pixels of CMOS sensors.

\section{Acknowledgments}

Alexandre Douplik gratefully acknowledges support of the Erlangen Graduate School in Advanced Optical Technologies (SAOT) by the German National Science Foundation (DFG) in the framework of the excellence initiative. This work was also supported in part by the Ryerson University Health Research Grant and the NSERC Personal Discovery Grant, Canada. The research was also partially supported by the Ontario Graduate Scholarship, Ryerson University, Toronto, Canada.

\section{References}

1. J. H. Takano et al., "Detecting early oral cancer: narrowband imaging system observation of the oral mucosa microvasculature," Int. J. Oral Maxillofac. Surg. 39, 208-213 (2010).

2. I. M. Braverman and A. Yen, "Ultrastructure of the capillary loops in the dermal papillae of psoriasis," J. Invest. Dermatol. 68(1), 53-60 (1977).

3. A. Yen and I. M. Braverman, "Ultrastructure of the human dermal microcirculation: the horizontal plexus of the papillary dermis," $J$. Invest. Dermatol. 66, 131-142 (1976).

4. I. M. Braverman and A. Yen, "Ultrastructure of the human dermal microcirculation: II. The capillary loops of the dermal papillae," $J$. Invest. Dermatol. 68(1), 44-52 (1977). 
5. R. A. Bennett, R. N. Pittman, and S. M. Sullivan, "Capillary spatial pattern and muscle fiber geometry in three hamster striated muscles," Am. J. Physiol. 260(2), H579-H585 (1991).

6. Y. Kumagai, M. Toi, and H. Inoue, "Dynamism of tumour vasculature in the early phase of cancer progression: outcomes from oesophageal cancer research," Lancet Oncol. 3(10), 604-610 (2002).

7. M. Cutolo et al., "Nailfold videocapillaroscopy assessment of microvascular damage in systemic sclerosis," J. Rheumatol. 27, 155160 (2000).

8. H.-Y. Li et al., "Current clinical applications of magnifying endoscopy with narrow band imaging in the stomach," Diagn. Ther. Endosc. 2012, 1-9 (2012).

9. A. J. Welch and M. J. C. Van Gemert, Optical-Thermal Response of Laser-Irradiated Tissue, Springer, London (2011).

10. A. Kienle et al., "Spatially resolved absolute diffuse reflectance measurements for noninvasive determination of the optical scattering and absorption coefficients of biological tissue," Appl. Opt. 35(13), 2304-2314 (1996).

11. D. A. Boas et al., "Imaging the body with diffuse optical tomography," IEEE Signal Process. Mag. 18(6), 57-75 (2001).

12. J.-M. Sainthillier et al., "Geometrical capillary network analysis," Skin Res. Technol. 9, 312-320 (2003).

13. R. Kiesslich et al., "New imaging techniques and opportunities in endoscopy," Nature Rev. Gastroenterol. Hepatol. 8, 547-553 (2011).

14. S. Marschall et al., "Optical coherence tomography-current technology and applications in clinical and biomedical research," Anal. Bioanal. Chem. 400(9), 2699-2720 (2011).

15. M. J. Pittet and R. Weissleder, "Intravital imaging," Cell 147, 983-991 (2011).

16. J. Allen and K. Howell, "Microvascular imaging: techniques and opportunities for clinical physiological measurements," Physiol. Meas. 35(7), R91-R141 (2014).

17. G. Saiko and A. Douplik, "Real-time optical monitoring of capillary grid spatial pattern in epithelium by spatially resolved diffuse reflectance probe," J. Innov. Opt. Health Sci. 05(02), 1250005 (2012).

18. E. M. C. Hillman and S. A. Burgess, "Sub-millimeter resolution 3D optical imaging of living tissue using laminar optical tomography," Laser Photonics Rev. 3(1-2), 159-179 (2009).

19. O. E. D. Online, "Oxford English Dictionary Online," Oxford English Dictionary, http://dictionary.oed.com (2010).

20. H. N. Chapman and K. A. Nugent, "Coherent lensless x-ray imaging," Nature Photonics 4, 833-839 (2010).

21. H. Jiang, G. Huang, and P. Wilford, "Multi-view in lensless compressive imaging," in Proc. 2013 Picture Coding Symposium (PCS 2013), pp. 41-44 (2013).

22. J. Spence, "Diffractive (lensless) imaging," in Science of Microscopy pp. 1196-1227, Springer, New York (2007).

23. O. Mudanyali, W. Bishara, and A. Ozcan, "Lensfree super-resolution holographic microscopy using wetting films on a chip," Opt. Express 19, 17378 (2011).

24. I. N. Papadopoulos et al., "High-resolution, lensless endoscope based on digital scanning through a multimode optical fiber," Biomed. Opt. Express 4(2), 260-270 (2013).

25. K. K. Hamamatsu Photonics, "FOP (fiber optic plates)," http://www. hamamatsu.com/us/en/product/new/3200/3031/index.html (accessed 12 September 2015).

26. K. Kretsos and G. B. Kasting, "A geometrical model of dermal capillary clearance," Math. Biosci. 208(2), 430-453 (2007).

27. F. Ayers et al., "Fabrication and characterization of silicone-based tissue phantoms with tunable optical properties in the visible and near infrared domain," Proc. SPIE 6870, 687007 (2008).

28. J. Pekar and M. S. Patterson, "Fabrication and characterization of phantoms with tissue-like optical properties from 500 to $700 \mathrm{~nm}$," Med. Laser Appl. 25, 147-153 (2010).
29. R. C. Chang et al., "Fabrication and characterization of a multilayered optical tissue model with embedded scattering microspheres in polymeric materials," Biomed. Opt. Express 3, 1326 (2012).

30. D. M. de Bruin et al., "Optical phantoms of varying geometry based on thin building blocks with controlled optical properties," J. Biomed. Opt. 15(2), 025001 (2010).

31. S. A. Prahl, M. J. C. van Gemert, and A. J. Welch, "Determining the optical properties of turbid mediaby using the adding-doubling method," Appl. Opt. 32(4), 559-568 (1993).

32. G. Saiko et al., "Optical detection of a capillary grid spatial pattern in epithelium by spatially resolved diffuse reflectance probe: Monte Carlo verification," IEEE J. Sel. Top. Quantum Electron. 20(2), 1-9 (2014).

Irina Schelkanova is an MSc and $\mathrm{PhD}$ student in the Physics Department of Ryerson University (Toronto, Canada). His area of research and interests covers a broad range of topics in photonics, biomedical optics, microcirculation, and cancer detection. Currently, he is working on the thesis: "Development of a Novel Miniature Fiber-Optics Technology for Quantification of Local Microvasculature Density Based on Hemoglobin Spatial Distribution."

Aditya Pandya is a PhD student at the Physics Department in Ryerson University (Toronto, Ontario, Canada). His areas of interest include novel variants of modeling light propagation in tissues using Monte Carlo (with GPUs, parallelized, and efficient approaches), surface enhanced Raman spectroscopy (SERS), nano- and biophotonics, cancer detection, data mining, machine learning algorithms, astrophotography, and gesture and voice recognition.

Guennadi Saiko obtained his MSc and PhD degrees and is currently associated with the Physics Department at Ryerson University. Prior to that, he worked in the Erlangen Graduate School in Advanced Optical Technologies at Friedrich-Alexander University ErlangenNuremberg, Erlangen, Germany, and before that at Ontario Cancer Institute, Toronto, Canada. His interests are related to biotissue optics and bioimaging with a primary focus on cancer detection.

Duoaud Shah is currently a project manager with the Techna Institute at the University Health Network in Toronto, Canada. He earned his MSc in medical biophysics from the University of Toronto, where his work was focused on designing, fabricating, and using microfluidic devices for single cell analysis.

Lothar Lilge obtained his education in physics from the WJ Goethe University in Germany, and from the West, Wilhelms University in Germany. His training included 3 years at Mass General Hospital, Boston, and 2 years at McMaster University Canada. He is a faculty in medical biophysics at the UofT and senior scientist at the PMH (Canada). His research interests are photodynamic therapy, optical spectroscopy for cancer risk assessment and development of tools for microfluidics.

Alexandre (Sasha) Douplik obtained his MSc, PhD, Habilitationsfach (Doctor of Science) and is an associate professor of the Physics Department at Ryerson Technical University and an affiliate scientist of Keenan Research Centre of the LKS Knowledge Institute at St. Michael Hospital, both in Toronto, Canada. He is a guest professor of the Erlangen Graduate School in Advanced Optical Technologies at Friedrich-Alexander University Erlangen-Nuremberg, Erlangen, Germany. His interests are related to the field of laser therapy/surgery and spectral/fluorescence bioimaging.

Biographies for the other authors are not available. 